\title{
Sistemas de organización del conocimiento: propuesta de un modelo unificado de definición
}

\author{
The knowledge organization systems: a unified model
}

\author{
Diego Ferreyra \\ Universidad de Buenos Aires. Facultad de Filosofia y \\ Letras / Centro Argentino de Información Cientifica y \\ Tecnológica (CAICYT-CONICET), Argentina \\ diego@r020.com.ar \\ (iD https://orcid.org/0000-0001-6823-5496
}

\section{Resumen:}

Este trabajo propone un modelo unificado para la definición de los sistemas de organización del conocimiento (SOC). Tras un recorrido a través de diferentes definiciones de SOC y las tipologías resultantes, se realiza un relevamiento de las debilidades teóricas señaladas en la bibliografía disponible. Se revisan las nociones de clasificación, organización del conocimiento y sistemas de organización del conocimiento. Se establece la necesidad de dar cuenta de las teorías del significado subyacentes en las concepciones de sistemas de organización del conocimiento. Con base a las críticas y los problemas relevados, se propone una definición de los sistemas de organización del conocimiento en tanto infraestructuras sociales de mediación simbólica diferenciados de otros dispositivos sociales por su carácter sistemático y formal. Asimismo, se establece la noción de autoridad cognitiva en tanto factor organizador del espectro de tipos de sistemas de organización del conocimiento. Finalmente, se ofrece un espectro de tipos de sistemas de organización del conocimiento resultante basado en el modelo propuesto.

Palabras ClaVE: Clasificación del conocimiento, Organización del conocimiento, Representación del conocimiento, Sistemas de organización del conocimiento, Infraestructuras del conocimiento.

\begin{abstract}
:
The aim of this paper is to propose a unified model for the definition of Knowledge Organization Systems (KOS). In the first place, we offer a survey of different definitions of KOS and the resulting typologies. We summarize what we consider certain theoretical weaknesses contained in the available bibliography, in order to point out that there is a need to draw attention to the different theories of meaning underlying in the different conceptions of KOS. Based on the criticisms and problems raised, a definition of $\mathrm{KOS}$ is proposed. In this new definition, KOS are understood as social infrastructures of symbolic mediation differentiated from other social devices due to their systematic and formal character. Likewise, the notion of cognitive authority is established as an organizing factor in the spectrum of types of systems for organizing knowledge. Finally, based on the model proposed, we offer a spectrum of types of KOS.
\end{abstract}

KEYWORDS: Classification of knowledge, Knowledge organization, Knowledge representation, Knowledge organization systems, Knowledge infrastructures.

\section{INTRODUCCIÓN}

El presente trabajo está centrado en un esfuerzo por componer un modelo unificado y coherente de los denominados sistemas de organización del conocimiento (SOC). A través del tiempo y la bibliografía se observan diversas conceptualizaciones con sus correspondientes esquemas de clasificación de dominio. Esta variedad expresa, por un lado, la diversidad de concepciones y el uso contemporáneo de nociones desarrolladas en sucesivos contextos socio técnicos; y por otro lado, da cuenta de tensiones teóricas y metodológicas no siempre formalizadas. Es posible considerar como un indicador de esta dispersión o falta de unidad orgánica en el dominio al uso contemporáneo de al menos cuatro expresiones: sistemas de

Recepción: 15 Enero 2021| Aceptación: 04 Mayo 2021 | Publicación: 01 Octubre 2021 
clasificación, lenguajes documentales, vocabularios controlados y sistemas de organización del conocimiento (Barité, 2011).

\section{LA ORGANIZACIÓN DEL CONOCIMIENTO}

Resulta sumamente llamativo, y a la vez alentador, que en un texto precursor y hasta inaugural del campo de la organización del conocimiento, Henry Bliss (1933) identifica algunos ejes de discusión que marcan fuertemente la agenda y las problemáticas de la clasificación de artefactos culturales. Quizás de manera amarga, Bliss (1933, p. 10) nota y lamenta que "hay bibliotecarios y educadores que desprecian el valor de la clasificación bibliotecaria. Sostienen que, siendo el conocimiento relativo y unitario, mientras que los intereses y los estudios son diversos e intrincados, las clasificaciones estructurales son igualmente relativas y las clasificaciones funcionales son transitorias; y que las formas de agrupar libros son ocasionales e indeterminadas. La mayoría de los usuarios de bibliotecas, dicen, tienen poca consideración por las clasificaciones, y la mayoría de las bibliotecas públicas las necesitan poco”. En esta cita, ${ }^{1}$ emerge tempranamente parte del entramado de tensiones que acompañan la problemática de los sistemas de organización del conocimiento: la tensión entre las modelizaciones formales del conocimiento y las prácticas sociales, la mutabilidad de las premisas de clasificación y los desajustes o divergencias entre las premisas de clasificación sociales en uso y las llamadas clasificaciones estructurales. Estas tensiones constituyen nuestro campo de estudio y trabajo, en una crítica a quienes pretenden abordar en tanto aspectos separados la clasificación del conocimiento de la clasificación de libros. El mismo Bliss (1933, p. 36) plantea que la tarea bibliotecaria está en el centro de esa tensión y considera que "existen dos nociones de clasificación, por un lado, la lógica, la natural y la científica, por otra parte, la práctica, la arbitraria y la intencionada; pero para la clasificación de bibliotecas debemos unir estas dos manos; los dos propósitos deben combinarse". ${ }^{2}$

En consonancia con la agenda universalista de su tiempo, este autor consideraba que la adopción de criterios de clasificación bibliográfica acordes a las premisas de organización del conocimiento de la ciencia y la educación habría de conllevar a una mejor resolución de las necesidades de búsqueda de los usuarios. No obstante, más allá de esto, resulta relevante notar para el presente trabajo, por un lado, la temprana conceptualización de la tensión entre los modelos institucionalizados del conocimiento y los usos sociales del conocimiento, y por el otro, la identificación de la biblioteca como el espacio que debe mediar o resolver esa tensión.

En línea con la percepción de que la organización del conocimiento constituye un fenómeno que desborda el campo de los vocabularios controlados, es quizás Dahlberg $(1978,1993)$ quien retoma esta agenda de manera sistemática para luego proponer la conformación de la organización del conocimiento en tanto espacio de investigación y prácticas. En la actualidad, es posible relevar importantes ejemplos de prácticas, metodologías, marcos teóricos y comunidades profesionales consolidadas en torno al campo de la organización del conocimiento, sin embargo, entre el conjunto de preguntas formuladas por el espacio de estudio, emergen algunas cuya resolución podría facilitar la adquisición y la construcción de herramientas teóricas y metodológicas. Entre esas dudas se identifican aquellas relativas a la dificultad para describir de manera orgánica, integral y coherente los SOC y su clasificación. Un indicador de esta dificultad puede observarse en la literatura académica a través de la preferencia por adoptar definiciones por extensión de los SOC. Se trata de definiciones conformadas por la enumeración del conjunto de elementos que conforman el universo de los SOC para cada autor, variando en algunos casos según dominio, función, estructura o contexto tecnológico.

Un segundo indicador de esta dificultad puede apreciarse en la apelación casi constante a retóricas gráficas para describir e identificar los atributos diferenciales entre los diversos tipos de SOC (véase el relevamiento y compilación de casos realizado por Souza, Tudhope y Almeida, 2012). Pareciera ser una estrategia que intenta dar cuenta del conjunto de tipos de SOC como un espacio relacional y orgánico sin necesidad de 
establecer un modelo coherente y constante de atributos diferenciales. Como señalan Souza et al. (2012), en muchos casos se apela a modelos analíticos unidimensionales que organizan el universo de los SOC como una progresión lineal de atributos o según el incremento gradual de algún rasgo o propiedad.

Un tercer indicador puede observarse en la debilidad de los acuerdos terminológicos acerca de la terminología en uso en el campo de los SOC (Mazzocchi, 2018). En algunas definiciones el etiquetado social (folksonomías) es un componente de los SOC (Hjørland, 2015; Kumbhar, 2012; Soergel, 2009), en otras queda excluido (Martínez Tamayo y Valdez, 2008), según sea el autor y el caso, los tesauros pueden ser considerados más estructurados que las taxonomías o viceversa (Mazzocchi, 2018; Souza et al., 2012). Se identifican enfoques que incluyen o excluyen alternativamente las herramientas de procesamiento de lenguaje natural, esquemas de metadatos, directorios de enlaces o la asignación de palabras claves sin control normalizado. Asimismo, la inclusión de las ontologías dentro de los SOC conlleva en algunos casos la eventualidad de analizar todos los vocabularios controlados como variantes de una clase mayor, la de las ontologías. Como señala Mazzocchi (2018), el universo de los vocabularios controlados es referido en algunos casos como un conjunto conformado por diferentes tipos de ontologías clasificadas según sean ontologías ligeras o complejas, el grado de fuerza semántica de sus descripciones, el grado de precisión ontológica o grado de axiomatización que permite. Esta diversidad terminológica expresa una debilidad en la definición del campo y la actividad.

Otro problema en relación con la definición de los SOC puede observarse en la dificultad para establecer cuál es el tipo apropiado para describir el universo de los SOC: ¿debe utilizarse un glosario? ¿Es la taxonomía la mejor estructura para formalizar el universo de los SOC o por el contrario, el límite de formalización axiomática que permite el esquema de relaciones léxicas del dominio son las listas? Si un SOC es una herramienta para la representación del conocimiento especializado, ¿cuál es el tipo de SOC que permite describir de manera adecuada sus tipos? ¿Cuáles son las relaciones léxicas y lógicas que vinculan entre sí a todos los términos que designan los SOC de manera orgánica, integral y total?

Finalmente, como señalan algunos autores (Hjørland, 2008; Mazzocchi, 2018), el campo de los SOC dispone de una práctica vigente y constante sin que exista una actividad teórica notoria o acorde a la magnitud de sus prácticas. Incluso resulta viable concluir que la elaboración de SOC emerge de manera espontánea en cualquier fenómeno de socialización (Eco, 2011; Leroi-Gourhan, 1971). La clasificación como actividad y los SOC en tanto dispositivos sociales resultan pues propios del medio social como tal y, al igual que la cultura o el lenguaje, su formalización teórica no es un requisito previo para su desarrollo y funcionamiento. No requieren de teorías, pero a través de ellas es posible establecer modelos analíticos que permitan disponer de mejores herramientas e intervenir de manera planificada en la institucionalización de las memorias sociales (Latour, 1998). Dicho esto, resultan infundados aquellos pronósticos, vaticinios y alarmas en relación al advenimiento de una era post-organización del conocimiento (Anderson, 2008; Hjørland, 2012; Mazzocchi, 2018; Weinberger, 2007). Los entornos como Google no constituyen una amenaza para los SOC, sino que son un caso de ellos. No existe tal cosa como una puja entre memorias sociales gobernadas por datos versus memorias sociales gobernadas por teorías (Anderson, 2008; Mazzocchi, 2018), ya que son instancias y aspectos de un mismo proceso y fenómeno.

\section{CLASIFICACIÓN Y ORgANIZACIÓN DEL CONOCIMIENTO}

En virtud de las problemáticas antes descritas, resulta de interés realizar un recorrido a través de las formalizaciones disponibles en la literatura especializada.

Son varios los autores (Barité, 2011; Hjørland, 2008; Martínez Tamayo y Valdez, 2008; Mazzocchi, 2018; Smiraglia, 2014) que reconocen en Bliss (1933) un antecedente en la conceptualización de la clasificación de materiales en bibliotecas como un fenómeno propio de la organización del conocimiento. Sostenía que si un libro es la materialización organizada del conocimiento y del pensamiento de un autor, la biblioteca puede 
ser vista como aquel entorno que reúne y sistematiza el conocimiento y el pensamiento de una comunidad. Descreído y contrario a las propuestas basadas en el ordenamiento temático según orden alfabético, Bliss creía que las bibliotecas debían disponer de un ordenamiento lógico-sistemático coherente con la que fuese la cosmovisión vigente y aceptada por una comunidad. Consideraba que dicha cosmovisión podía observarse en el sistema científico o educativo de una sociedad. Sus propuestas y puntos de vista expresan de manera temprana algunos problemas que luego fueron estudiados por la bibliotecología y otras disciplinas.

Tanto Bliss como otros autores contemporáneos, merced a la idea de una unidad orgánica del conocimiento universal, veían al libro y al documento como una manifestación, un accidente materializado del conocimiento, y a este último como el verdadero centro, finalidad y objeto de estudio. Esta concepción del campo bibliotecológico (y de la documentación) cobró nuevo impulso ante la creciente intrascendencia de los soportes materiales de los artefactos culturales, la generalización de los entornos digitales en red y la convergencia tecnológica de los procesos de mediación cultural.

Es posible considerar que la emergencia y la aplicación de herramientas informáticas a la gestión bibliotecaria y el desarrollo incipiente, pero sostenido, de las ciencias cognitivas durante la segunda mitad del siglo XX establecieron las condiciones de posibilidad para la formalización de propuestas en el campo de la bibliotecología y la documentación. Que asimismo, desplazaron los ejes disciplinares enfocados en los soportes materiales (libros, documentos y otros artefactos) hacia el estudio de la cognición, los procesos semióticos y la mediación documental como singularidad (Dahlberg, 1978; Licklider, 1965; Martín Serrano, 1977; Wilson, 1983). En ese contexto puede situarse el trabajo de Dahlberg (1978, 1993, 2006) orientado a consolidar y formalizar un dominio dedicado al conocimiento en tanto entidad cognitiva y humana y su dinámica particular en los procesos de búsqueda. Sus definiciones y acercamientos hacia el conocimiento como entidad mental, la formalización sistemática del conocimiento en tanto estructuración coherente y cohesiva de relaciones y su impacto en la representación y organización del conocimiento delimitaron una agenda y un campo de trabajo productivo y fértil dotado de problemáticas propias, capaz de incorporar modelos teóricos y dar cuenta de fenómenos presentes y pasados.

Las definiciones de dominio propuestas por Dahlberg (2006) suponen la incorporación de, al menos, tres supuestos críticos y transformadores. En principio, la consideración del conocimiento como entidad cognitiva; en segundo lugar, la necesidad de un modelo semiótico capaz de dar cuenta de la dinámica específica de los procesos de la búsqueda y de la mediación documental; y finalmente, en tercer lugar, la implicancia mutua de los dispositivos de representación del conocimiento y los de organización del conocimiento. Estas asunciones abrieron el campo teórico y metodológico hacia la terminología, la lingüística, la semiótica y la lógica entre otras disciplinas. Asimismo, ampliaron el campo de acción antes limitado a los sistemas de clasificación documental hacia los glosarios, las ontologías, los diccionarios, las listas de valores y otros dispositivos de representación del conocimiento.

Para finales del siglo XX, la centralidad de los procesos de representación del conocimiento y la conceptualización de los mismos en tanto meta-dispositivos (Bowker y Star, 2000; Smiraglia, 2014) capaces de proveer portabilidad y autonomía a estructuras simbólicas de todo tipo -desde cosmovisiones universales hasta modelos de atributos para la caracterización de procesos puntuales- se constituyó en una necesidad social que atravesaba todas las actividades. Es así como, otras disciplinas comienzan a estudiar los problemas de la clasificación con sus propios modelos teóricos y metodologías. En el campo de la bibliotecología y la documentación, el borde expuesto hacia esa creciente demanda de soluciones descentradas de las condiciones materiales de los soportes fue tomado por la agenda de las llamadas bibliotecas digitales. Es así que en un informe encomendado por la Digital Library Federation, una organización cooperativa surgida a fines del siglo XX, Hodge (2000) ofrece una de las definiciones más difundidas y utilizadas de la noción de SOC. La autora analiza la pervivencia y la vigencia aún en contextos digitales de una serie de herramientas ya existentes y reunidas bajo la denominación común de sistemas de organización del conocimiento ("knowledge organizing systems") ${ }^{3}$. El informe establece que los SOC son el corazón mismo de las bibliotecas, museos 
y archivos. Dicho centro vital comprende los esquemas de clasificación, los encabezados de materias, los diccionarios lexicales, diccionarios geográficos (gazetteers), los sistemas de gestión de autoridades de todo tipo, los tesauros y taxonomías, las redes semánticas y las ontologías. Es posible notar en la enumeración (definición por extensión) el esfuerzo teórico por articular las herramientas tradicionales del campo con los emergentes propios de las infraestructuras digitales de gestión documental.

Si bien el informe presenta las mencionadas herramientas en tanto el corazón de las bibliotecas, museos y archivos, extiende su uso hacia cualquier soporte digital o basado en la web. Hodge (2000) considera que la función principal de los SOC es dar soporte a los procesos de búsqueda y recuperación en entornos digitales.

Con base en la productividad un tanto polivalente de la formalización de Dahlberg (2006) y la vaguedad enumerativa de Hodge (2000), se despliegan sucesivas y diversas delimitaciones y enfoques para los SOC. Zeng (2008) propone clasificar los SOC según las principales funciones y características estructurales prevalentes y jerarquizados siguiendo un orden creciente de complejidad. Soergel (2009) define los SOC como una infraestructura conceptual que interviene en todo tipo de procesos de categorización y organización de recursos que pueden ser clasificados según sus orígenes, funciones, estructura, presentación o dinámica de elaboración entre otros criterios. Barité (2011) identifica como rasgos esenciales de los SOC a la referencia a un conocimiento especializado, la estructuración lógica de las relaciones entre los términos y la adopción de pautas de control para la elaboración y selección de términos. Asimismo, diferencia 13 tipos de SOC según criterios de organización interna, tipo de relación con los recursos indizados o modalidades de representación del conocimiento. Martínez Tamayo y Valdez (2008) igualan el alcance de los SOC al de los vocabularios controlados en tanto lenguajes artificiales utilizados para clasificar e indizar.

Desde una perspectiva que se aproxima a los estudios del lenguaje, Hjørland (2007) entiende que, en definitiva, los instrumentos mencionados por Hodge refieren en todos los casos a herramientas semánticas que pueden ser diferenciadas y clasificadas según el tipo de relaciones semánticas establecidas entre las unidades léxicas que los componen. De esta manera el autor establece que todo SOC es en definitiva una herramienta semántica. Se observa aquí una noción de SOC que cobra cierta autonomía en relación con la operatividad funcional de la búsqueda o representación de recursos. Se fortalece pues una concepción de los SOC en tanto constructos que modelizan y dan cuenta de cosmovisiones. De manera quizás aún más potente Hjørland (2015) considerará que todo SOC es en sí mismo una teoría del mundo.

Wright (2008) presenta varias propuestas de organización del universo de los SOC, si bien no ofrece una definición operativa ni sistemática, en un trabajo que destaca por la amplitud de casos contemplados. La autora establece una primera discriminación según el tipo de relaciones semánticas entre los elementos léxicos que componen cada SOC, caracterizando las mencionadas relaciones semánticas según su grado de sistematicidad (sistemáticas, no-sistemáticas, híbridas). Paralelamente caracteriza los mismos SOC según sus usos, funciones, dominios de origen y comunidades de prácticas adoptantes.

Como punto final de este recorrido es posible mencionar la revisión y análisis de las tipologías de SOC realizado por Lara (2015). A través de una noción amplia de SOC en tanto aquellos instrumentos dedicados al "contenido" de los documentos con miras a facilitar la gestión de los mismos, la autora considera que las actuales tipologías resultan deficientes y limitadas. Sostiene que las mismas no logran dar cuenta de la diversidad de instrumentos en uso. Entre las debilidades identificadas por Lara (2015) en la teorización de los SOC, resulta de interés remarcar dos de ellas. En primer término la autora llama la atención sobre la apelación a modelos lógicos tradicionales para la caracterización de las estructuras internas de los SOC y las limitaciones de este enfoque dar cuenta de la diversidad de instrumentos en uso. En segundo lugar, remarca las problemáticas implicadas en la representación del conocimiento social a partir las dificultades metodológicas para analizar las condiciones de autoridad discursiva de los SOC en sus dinámicas de elaboración y utilización.

Resulta interesante observar que parece existir un consenso claro acerca de la incidencia del contexto (en cualquiera de sus dimensiones) en los procesos de representación del conocimiento. Sin embargo, en relación con la organización del conocimiento, perviven enfoques que conciben la gestión del conocimiento como un 
proceso neutral, transparente y potencialmente universal (o libre de contexto). Este tipo de disyunción entre dos procesos que serían articulares y mutuamente dependientes impacta necesariamente en las concepciones de SOC resultantes. Podría decirse que resulta más sencillo reflexionar acerca de los supuestos ideológicos en el plano de la representación, antes que intervenir y decidir en las manifestaciones y consecuencias de los procesos de gestión que operan y organizan la materialidad de la vida cotidiana.

\section{LOS SISTEMAS DE ORGANIZACIÓN DEL CONOCIMIENTO}

Como ha podido apreciarse, mayormente las aproximaciones a una definición de los SOC se caracterizan por definiciones por extensión o enumeración de casos (Barité, 2011; Hodge, 2000; Suárez Sánchez, 2017). Este tipo de enfoque, característico de los momentos de consolidación de nuevos espacios de estudio, presenta en la actualidad serias limitaciones para su crecimiento y expansión. Primeramente no parece existir un cuerpo estable, acotado y económico de criterios capaces de sistematizar una tipología de SOC. En segundo lugar, se presentan escollos teóricos y metodológicos para incorporar las innovaciones y variaciones que se van presentando día a día o los SOC elaborados y utilizados en otros contextos históricos o socioculturales.

De manera liminar resulta necesario establecer un marco de referencia que defina a los fines del presente trabajo la noción en uso de SOC, la clasificación como fenómeno y la indización en tanto actividad. En principio y desde un punto de vista amplio, se considerará en tanto clasificación toda operación capaz de segmentar o establecer una discontinuidad sobre la continuidad de la experiencia del mundo (Bowker y Star, 2000). Ya sea distinguir la luz de la oscuridad, separar el mar de los cielos o la organización de la botánica terrestre en especies, en todos los casos se trata de segmentar y diferenciar el mundo a través de una experiencia y una cosmovisión humana.

En la multitud de fenómenos de clasificación disponibles, desde el campo disciplinar de la bibliotecología, la clasificación es considerada como un proceso de mediación institucional, se trata de procesos sistemáticos y formales de clasificación de recursos en relación con propósitos explícitos. La clasificación, para nuestro campo, es un tipo de mediación simbólica basada en la asignación sistemática de categorías intencionales a determinados recursos. Esta definición permite diferenciar las prácticas de clasificación en el contexto disciplinar de la bibliotecología de otras prácticas de categorización en función de su especificidad intencional y del carácter sistemático implicado en sus procesos de diseño, gestión, mantenimiento y gobierno (Glushko, 2014).

Dicho lo anterior, es posible establecer que el conjunto de elementos, herramientas y métodos que conforman los procesos de clasificación orientados a dar soporte a procesos de mediación entre recursos y sujetos sociales integran los SOC. Se trata de dispositivos de clasificación de carácter intencional, sistemático y formal. Son deliberados en tanto surgen de procesos mentales, ya sean reales o modelizados en un esquema o algoritmo. La noción de intencional refiere a que la clasificación surge de un proceso mental (o emergente a partir de la relación entre la mente y el mundo), no como atributo físico de los recursos. Se trata de dispositivos sistemáticos ya que, idealmente, proceden de manera regular y predecible: clasifican siempre de la misma manera. Y finalmente son infraestructuras formales en tanto la intencionalidad y la sistematicidad se encuentran explicitadas en medios y dispositivos específicos. Pueden ser tablas, algoritmos o políticas de clasificación, pero si un ser ajeno a todo lo humano, por ejemplo un extraterrestre, analizara un SOC y las prácticas de clasificación asociadas, podría reconstruir la subjetividad a través de la sistematicidad y regularidad codificada en él. Esta regularidad no procede de la misma manera en todos los contextos (Glushko, Maglio, Matiock \& Barsaiou, 2008), la organización personal y privada de nuestros libros, listas de reproducción de música o las carpetas de correo electrónico sigue pautas de regularidad y dinámicas muy diferentes. En la vida privada se guarda un objeto intentando imaginar dónde se lo buscará en el futuro, es decir, se efectúa una modelización informal de nuestra subjetividad futura. 
Por su parte, Romanos de Tiratel (1993) propone considerar la profesión bibliotecaria como una disciplina signada por el diseo y la implementación de procesos de comunicación potenciales y diferidos. La clasificación de recursos en el contexto de las prácticas bibliotecológicas también implica la formalización y la modelización de un sujeto social a través de una definición establecida y explícita. Cuando se ordena un libro en una biblioteca infantil no se lo hace según nuestros gustos literarios, sino según la modelización cognitiva disponible acerca de los nios que utilizan la biblioteca. El mismo libro en una biblioteca orientada a investigadores sobre literatura infantil probablemente sería clasificado de otra manera.

Los SOC proceden a través de la vinculación de un material lingístico (un término o palabra), un sujeto (real o modelizado) y un recurso (documento, objeto o incluso un concepto). La asignación de términos a un recurso también es conocida en el campo de la bibliotecología y ciencias de la información como indización. Se trata de un proceso orientado a generar una representación lingística de los temas de un documento (Lancaster, 1998).

En los anteriores apartados se caracterizó la clasificación como proceso cognitivo y social, resulta ahora necesario abordar los SOC en tanto dispositivo sociotécnico. Dahlberg (2006) en su camino hacia la formalización de la OC como campo, opta por analizar la composición conceptual del término. La autora define organización como la acción de construir algo de acuerdo a un plan y concibe al conocimiento como un fenómeno restringido exclusivamente a la dimensión humana y caracterizado por la posibilidad de compartir información socialmente. A través de esta concepción, el conocimiento es un fenómeno humano susceptible de ser compartido o socializado con un propósito. En estos términos, el conocimiento es un fenómeno tanto humano como social. No puede existir por sí solo en la mente de un humano, sino que existe en tanto material social en circulación. Este desplazamiento de recursos simbólicos a través del tiempo y el espacio, procede a través del lenguaje. En estos términos, el conocimiento es todo aquello que puede ser transmitido por fuera de la biología, utilizando el lenguaje como infraestructura primaria.

Aún resta analizar la noción de sistema. En el contexto de los SOC, se trata de dispositivos previstos, determinados y caracterizados en sí por procesos específicos. Es decir, no surgen de manera espontánea o por inercia, sino que han sido previstos por alguien (un actor o agencia de cualquier tipo). Asimismo, son determinados en tanto disponen de límites y requieren de la definición formal de las entidades que los conforman y de las relaciones posibles en el contexto del sistema. La dinámica de interacción entre elementos y relaciones habrá de definir los procesos que caracterizan al sistema como tal.

En síntesis, los SOC son dispositivos de mediación social que operan a través de la formalización sistemática de representaciones. Esta definición aún encierra algunos problemas, puntualmente desplaza parte de la elusividad de las definiciones antes mencionadas a la definición de sistematicidad y formalización. ¿Cuál es el régimen de esta sistematicidad? ¿Cuál es su regularidad y su invariabilidad? ¿Cómo procede la formalización?

Por último, es preciso analizar la vinculación entre los SOC y la clasificación. La clasificación entendida como la segmentación de la experiencia, como el proceso de diferenciación e identificación del mundo a través de nuestras concepciones y percepciones, es una expresión de la forma en que procede nuestro conocimiento como sujetos. Los esquemas de clasificación utilizados por una comunidad constituyen una formalización accesible de lo que esa comunidad entiende y considera en tanto conocimiento en un contexto dado. Por ejemplo, si al ingresar a una escuela se nos pregunta por nuestra edad para luego asignarnos un aula y una determinada currícula de contenidos, esto permite entender aspectos de la cosmovisión de esa comunidad: en ella la edad es un atributo determinante para la asignación de aprendizajes y conocimientos.

Si la clasificación constituye el núcleo teórico y metodológico de los SOC, la indización puede considerarse como su accionar operativo. 


\section{UNA TEORÍA DEL SIGNIFICADO DE LOS PROCESOS DE BÚSQUEDA}

Establecido la centralidad de los procesos de representación y mediación en la clasificación, queda claro que una teorización acerca de la organización del conocimiento y la tipificación de los SOC requiere una teoría del sentido, quizás incluso una teoría del sentido orientada a la búsqueda como proceso. En una línea de pensamiento cercana, Blair (1990, p. 122) sostiene que "el proceso de representación de documentos para su recuperación es fundamentalmente un proceso lingüístico. Por lo tanto, cualquier teoría de la indización o representación de documentos presupone una teoría del lenguaje y el significado". ${ }^{4}$ De acuerdo con Blair, no es posible establecer un modelo de mediación documental sin una teoría del significado, todo SOC dispone e implica una particular teoría del sentido. Con base en lo antedicho, se observa que una teoría de la indización o de la representación de recursos -tal como la describe Lancaster (1998)-supone el desarrollo de una teoría del significado que sea capaz de definir un régimen de relación entre tres entidades diferenciadas: una conceptualización del recurso u objeto a describir, una concepción o definición del sujeto y una concepción del signo. Esta relación fue esquematizada en sucesivos triángulos por diversas teorías del significado.

FIGURA 1

Triángulo de Ogden y Richards o Triángulo semiótico

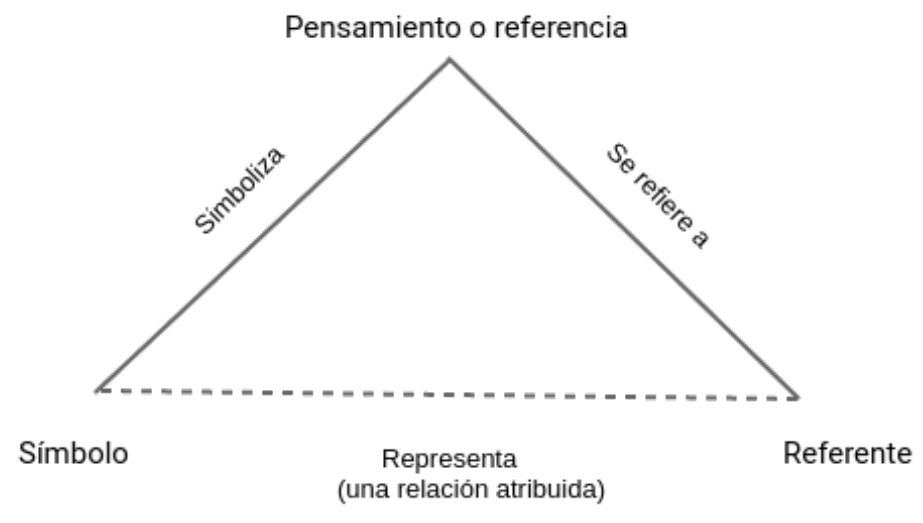

Fuente: elaboración propia basada en Ogden y Richards (1964, p. 29).

En la figura 1 se presenta el esquema elaborado por Ogden y Richards (1964) y abordado por muchos otros enfoques (Almeida, Souza \& Fonseca, 2011; Dahlberg, 1978; Hjørland, 2007) a la hora de establecer una teoría de la significación o del significado como proceso. Aquí la significación se describe como una relación ternaria, analizable en tres relaciones binarias entre los vértices, dos básicas y una derivada (en línea punteada). De esta manera la relación entre un símbolo $($ symbol) y su referente (referent) estaría mediada por un concepto (thought or reference).

La figura 2 muestra la síntesis comparativa elaborada por Eco (1994, p. 26) de los términos utilizados por lingüísticas y filósofos para dar cuenta de la convergencia entre concepciones del signo. 
FIGURA 2

Triángulo que contempla los términos utilizados por Pierce, OgdenRichards, Frege, Carnap, Morris, Saussure, Sturart Mill, Hjekmslev, Buyssens.

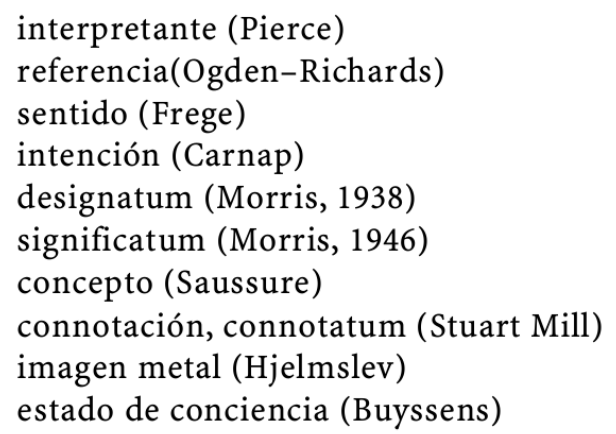

Fuente: Eco (1994, p. 26).

La centralidad de una teoría del significado no resulta nueva para nuestro campo y fue la misma Dahlberg (1978) quien la consideró fundamental para la viabilidad de la organización del conocimiento como dominio la definición de las nociones de concepto, término y referente y el régimen de relaciones posibles entre estas entidades. La autora parte de las aproximaciones hacia la semiótica realizadas por el sociólogo Sartori (1984) y en tal sentido define la noción de concepto en tanto unidad de conocimiento, siendo esta unidad comprendida por el conjunto de declaraciones verificables sobre un elemento de referencia específico (referente de la realidad) representado en forma verbal (unidad léxica, término). La autora adopta y adapta el triángulo semiótico elaborado por Sartori (1984) y establece su utilidad para dar cuenta de los procesos de organización del conocimiento. Con el fin de ponderar la vigencia de este análisis, vale realizar algunas aclaraciones referidas a la noción de referente y unidad del conocimiento de Dahlberg (1978).

El texto considera que un referente puede ser un objeto, un conjunto de objetos considerados como una unidad, una propiedad, una acción, dimensión, es decir, cualquier entidad concreta o abstracta (o combinación de los elementos antes mencionados). Asimismo, define concepto en tanto el conjunto de declaraciones verificables sobre un elemento de referencia seleccionado, representado en forma verbal. Se observan aquí los tres elementos y su respectivo régimen de vinculación. Sin embargo, tal como está planteada, esta sería una teoría del conocimiento analítico lógico, no necesariamente del conocimiento simbólico. Es posible decir que, todo conocimiento lógico es simbólico, pero no todo conocimiento simbólico cumple necesariamente con un conjunto de premisas lógicas previas. Para que esta definición pueda abarcar las representaciones sociocognitivas, en general, resultaría apropiado reemplazar la condición de declaración verificable por declaración atribuible. Una declaración atribuible sería cualquier enunciado susceptible de ser atribuido (o predicado) por un agente a un referente. En la figura 3 se presenta el triángulo semiótico tal como fue presentado por el autor. 
Triángulo semiótico presentado por Dahlberg (1978).

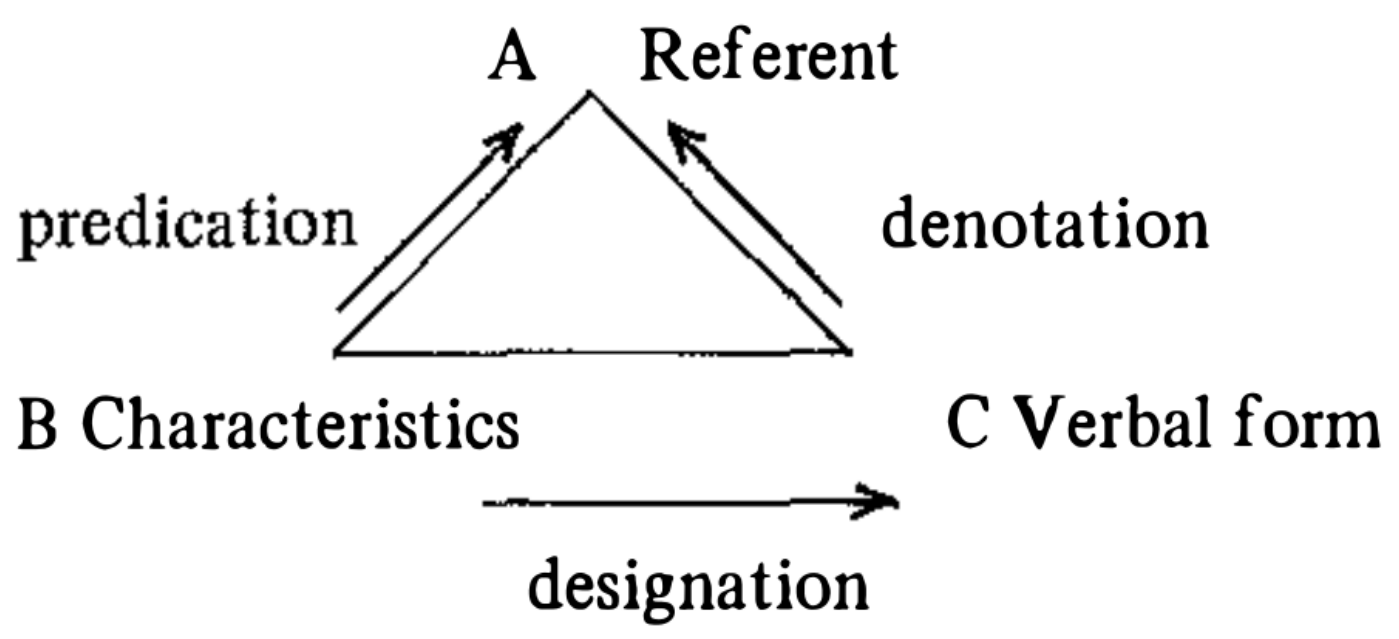

Fuente: Dahlberg (1978).

Hjørland (2007) también considera que la noción de significado constituye un eje central y organizador del campo de los SOC. El autor sostiene que existe una concepción amplia de SOC y una restringida. La amplia contempla diferentes prácticas vinculadas a la organización de lo social (la organización de literaturas, tradiciones, disciplinas, enciclopedias, bibliotecas o bases de datos) en tanto la noción restringida estaría definida por las herramientas específicamente desarrolladas para la organización de repertorios bibliográficos. A través de esta distinción los SOC destinados a la organización de repertorios bibliográficos serían un subconjunto dentro de un conjunto mayor. Según Hjørland (2007) el rasgo diferencial de los SOC en sentido estricto no sería sólo su campo de aplicación sino también su especificidad en tanto herramientas semánticas dedicadas a la definición y enriquecimiento de relaciones conceptuales. La propuesta de Hjørland (2007) constituye un esfuerzo por ampliar el campo de trabajo del marco de los SOC más allá de las fronteras del espacio documental.

\section{DiNÁMiCa de los SISTEMAS DE ORgANizACión DEL CONOCIMIENTO}

$\mathrm{Si}$, como se ha observado anteriormente, los SOC son un instrumento, una herramienta, una infraestructura, por lo que ha llegado el momento de describir su dinámica operativa. ¿Cómo proceden los SOC? ¿Cuál es el impulso por el cual todos los entornos sociales construyen SOC?

Cuando un sujeto delega en un sistema, un entorno, un esquema de clasificación, un especialista o un amigo (o cualquier tipo de agente en términos de actor de una acción) la facultad o tarea de categorizar o clasificar un evento o fenómeno, ese sujeto confía y asigna un estatus de autoridad cognitiva al agente de categorización. La autoridad cognitiva es la posibilidad de confiar en un conocimiento que no se origina en la propia experiencia personal (Goldman, 2010; Wilson, 1983). Un SOC está provisto de autoridad cognitiva cuando se confía en su capacidad para clasificar sin la necesidad de ser nosotros mismos quienes lo hacemos. Ocurre cuando un crítico comenta una película, un amigo recomienda un destino de vacaciones basado en su experiencia directa o cuando una institución certifica la validez de un medicamento. Es parte de la socialización del conocimiento, constituye un fenómeno cotidiano y constante en la vida social derivado de la imposibilidad de disponer de vivencias directas o testimonios primeros de todas las experiencias posibles. 
Eventualmente la noción de autoridad cognitiva puede ser analizada desde otras categorías, como autoridad epistemológica o regímenes de verdad (Foucault, 2014). Si bien no son categorías equivalentes, en el presente artículo se optó por preferir la noción de autoridad cognitiva en virtud de su capacidad de diálogo con modelos orientados a analizar la mediación simbólica en tanto proceso cognitivo. La noción de autoridad cognitiva es una percepción socialmente construida, es dinámica y no uniforme (Wilson, 1991). Una entidad puede ser considerada como autoridad cognitiva para un momento, para un tema o en determinado contexto y no en otro. La potencia de la conceptualización desarrollada por Wilson (1983) surge de la sencillez con la que aborda el tema. El autor sostiene que en la mayoría de nuestros eventos vitales es necesario recurrir a otros para disponer de ideas o información acerca de cosas que están fuera del rango de nuestra experiencia directa. Es así que en un entorno social se depende mayormente de criterios y percepciones de segunda mano, necesariamente hay obligación de suspender temporalmente nuestro juicio crítico, delegar criterios y confiar en la experiencia y conocimiento de otros. El autor comienza por preguntarse cómo la gente decide quién sabe y sobre qué. Clasificar implica operar a través de criterios, un SOC cristaliza y estructura estos criterios de manera sistemática. Cuando se define si una persona con la etiqueta "compañero de estudios", los contenidos asociados a esa persona quedarán vinculados a un conjunto de contenidos de manera estable y sistemática. Utilizar un SOC implica delegar momentáneamente juicios y criterios en un sistema. Toda vez que se recurre a un esquema de mediación basado en algún tipo de clasificación que opera de manera sistemática, modelizada y potencialmente previsible, se está ante un SOC y se ha delegado en el sistema algún grado de autoridad cognitiva.

Hasta aquí se ha propuesto una definición de los SOC y se ha establecido su dinámica de operación. Surge una vez más la pregunta: ¿Cómo es que delegamos autoridad cognitiva en un sistema? ¿Qué regímenes de verdad operan en cada SOC? ¿Todos los SOC apelan al mismo régimen de verdad?

\section{TIPOS DE SOC SEGÚN ESQUEMA DE AUTORIDAD COGNITIVA}

Tanto la representación como la organización del conocimiento son procesos basados en la mediación simbólica, resulta útil pues recurrir a la noción ternaria de proceso de significación utilizada ya por diversos enfoques desde el siglo II, ${ }^{5}$ y esquematizada por Ogden y Richard (1964). Se adoptará esta propuesta considerando su capacidad para dar cuenta de las entidades involucradas en los procesos de búsqueda: un agente cognitivo e interpretante, una materialidad simbólica (en nuestro caso, lingüística) y un recurso o referente. Esta correspondencia esquemática no se condice necesariamente con todas las condiciones establecidas por los distintos autores que analizaron o utilizaron la concepción ternaria del significado. A los fines de la presente indagación no se aborda la discusión acerca de la dinámica de relación entre los tres componentes. Es interés de este estudio centrarse en esta estructura triádica para establecer una correspondencia que permita clasificar los SOC según las estrategias de delegación de autoridad cognitiva a las que apela cada uno de ellos.

Se propone, pues, una clasificación de los tipos de SOC en base a uno de los aspectos estructurales de los mismos: los procesos de mediación y apropiación de recursos basados en lenguaje. Estos procesos de asignación de atributos pueden ser definidos y diferenciados según el abordaje y rol asignado a cada uno de los siguientes elementos:

1. el objeto o recurso a representar (referente).

2. el interpretante (modelizado o real).

3. el lenguaje (símbolo). 
Esto plantea una composición tripartita: tres fuentes que regulan las condiciones de legitimidad de los procesos de mediación de manera articular. En el tipo y la dinámica de modulación entre estas fuentes se define el ciclo de vida y la eventual eficacia de cada SOC.

Si la clasificación es una actividad que establece una relación entre un sistema de signos (L), un sujeto social modelizado en un interpretante (I) y un referente o recurso (R), los SOC pueden ser discriminados según consideren a cada uno de los componentes de la relación tripartita en tanto condición prevalente de condiciones de autoridad cognitiva.

Se consideran los SOC orientados a obtener legitimidad en base a su fidelidad en relación al referente (R). Se considera que los mismos están centrados en la extracción de elementos del mismo referente, por ejemplo, extracción de palabras clave, identificación y extracción de entidades (nombres, lugares, fechas, etc) u otras estrategias basadas en la fidelidad e identidad con respecto al referente. Este tipo de esquemas depende de manera primaria del referente. Es el momento de comenzar a responder una de las preguntas relativas a las restricciones sistemáticas implicadas en esta definición. Los SOC centrados en el referente requieren que el referente preexista al SOC, es condición de posibilidad de los mismos la existencia previa del recurso que será analizado. Resulta imposible modelizar u operar este tipo de SOC sin la disponibilidad de referentes para su análisis y clasificación.

Los SOC basados en la indexación de textos completos apelan en última instancia a la premisa de objetividad del referente. La existencia de modelos que supeditan sus condiciones de legitimidad, régimen de verdad o autoridad cognitiva en la noción de objetividad, identidad o fidelidad con respecto a un determinado referente, no implica en ningún sentido por nuestra parte la asunción de que no existe una modelización mediadora entre todo referente y su representación. Un aspecto saliente y diferencial de las retóricas de legitimidad asociadas a esta estrategia puede identificarse en su apelación a discursos de transparencia y neutralidad.

Estos sistemas encarnan estrategias de indización que apelan a la premisa de objetividad: "La objetividad preserva el artefacto o la variación que se habría borrado en nombre de la verdad; tiene el escrúpulo de filtrar el ruido que socava la certeza. Ser objetivo es aspirar a un conocimiento que no deje rastro del conocedor, un conocimiento que no esté marcado por los prejuicios o la habilidad, la fantasía o el juicio, el deseo o el esfuerzo" (Daston y Galison, 2007). ${ }^{6}$

Encuentran entornos favorables en contextos en los que se disponen corpus textuales no mensurables o con dinámicas de crecimiento fuera de escala de procesamiento humano (como es el caso de la web) o contextos en los que existe fuerte identificación entre la cosmovisión de los usuarios y la materialidad textual de los recursos (por ejemplo algunos repositorios científicos). El explorador cromático de Google, ${ }^{7}$ se presenta como un ejemplo claro de este tipo de SOC que busca cuadros en los que predomine el color seleccionado sin ponderar el eventual significado compositivo de cada color.

Ha llegado el momento de abordar aquellos SOC que obtienen sus condiciones de autoridad y legitimidad con base en su capacidad para representar la singularidad cognitiva del sujeto (I). Estos dependen de manera primaria de las representaciones terminológicas generadas por los propios usuarios. En este caso la restricción sistémica se encuentra definida por la preexistencia del sujeto, resulta imposible operar este tipo de SOC sin la acción del agente activo de clasificación. En algunos trabajos se propone diferenciar este tipo de SOC por su carácter colaborativo, sin embargo, los SOC no resultan ajenos a las dinámicas de colaboración. Una ontología, un categorizador textual o cualquier tipo de SOC puede ser el resultado de un trabajo colaborativo. El carácter participativo por sí mismo no constituye pues una restricción sistémica para caracterizar un tipo de SOC. Lo que distingue este tipo de SOC es el modelo de intervención de esa colaboración en relación con los procesos de producción de significado.

En este tipo de sistemas el grado de legitimidad de cada indización no procede de su nivel de objetividad (entendido como fidelidad e identidad a un objeto), sino por la frecuencia con la que un sujeto utilizó la misma formalización terminológica para vincular el mismo recurso. Este tipo de asignación de palabras 
clave sigue la dinámica propia de la construcción de conceptos cotidianos: se elaboran y desarrollan en un momento particular y específico. Se encuentran determinados por el contexto de emergencia (Luria, 1984). La autoridad cognitiva de este tipo de SOC está directamente asociada a las clasificaciones realizadas activamente por los sujetos.

En estos SOC el esquema triádico del significado encuentra su correlato más explícito: un sujeto interpretante (I) vincula un conjunto de términos (L) con un recurso en tanto referente (R). Resultan ser los SOC más dinámicos y dependientes del contexto: para operar requieren que preexistan tanto los recursos que serán referidos como el sujeto social que habrá de asignarles un término o etiqueta. Las herramientas de etiquetado social (tags, etiquetas) o folksonomías son ejemplos clásicos de este tipo de SOC. El uso de algoritmos entrenables en base a las elecciones de los usuarios constituye ejemplos híbridos que utilizan las elecciones y las clasificaciones realizadas por los usuarios para enriquecer y moderar indizaciones previamente realizadas con base en el procesamiento de texto completo.

Estos sistemas encuentran entornos favorables en ambientes que obtienen un plusvalor a través del reconocimiento de identidades y cosmovisiones (mediación social), en el contexto de las redes sociales en las que el identificación de afinidades resulta la forma prevalente de búsqueda y exploración. Este tipo de SOC opera con mayor facilidad en contextos de comunidades emergentes o inestables o dominios que no dispongan de modelos sistemáticos o consensuados de organización.

Finalmente ha llegado el momento de los tipos de clasificación basados en los modelos de autoridad provistos por las relaciones intrínsecas del sistema de representación utilizado. Se trata de SOC que apelan a la construcción de una red de coherencia semántica a través de las relaciones léxico-semánticas del lenguaje. La potencia del régimen de verdad o autoridad cognitiva deriva en este caso de la capacidad para operar y someter un dominio a las condiciones de coherencia establecidas en las premisas léxico-semánticas del SOC. Esta podría ser la razón por la cual la definición de ontología solapa potencialmente el campo de los demás SOC que aluden a este modelo de autoridad. Si un tesauro refiere a un número acotado y fijo de premisas de coherencia (principio del tercero excluido, principio de identidad, etc.), una ontología permite definir de manera puntual, local y dinámica las premisas que gobiernan su red de coherencias. La posibilidad de definir formalmente las excepciones del modelo de red de coherencias es quizás una de las principales promesas y fortalezas diferenciales de las ontologías.

Cabe señalar que, en cada caso, el SOC da por aceptado que la red de relaciones de coherencia surge de vinculaciones de sentido propias del lenguaje y de su estructura de asignación de significado. Por ejemplo, los tesauros asumen que las relaciones jerárquicas (hiperonímia/hiponímia/meronimia), asociativas, de sinonimia u otro tipo de relaciones léxico-semánticas pueden ser analizadas como un modelo de relaciones coherentes y definido en el lenguaje. Son el tipo de SOC con mayor desarrollo en el campo bibliotecológico y sobre el cual existe una experiencia vasta.

En el ensayo "El ruiseñor de Keats", Jorge Luis Borges propone una división según la forma de representar la relación entre orden y mundo: "Observa Coleridge que todos los hombres nacen aristotélicos o platónicos. Los últimos sienten que las clases, los órdenes y los géneros son realidades; los primeros, que son generalizaciones; para estos, el lenguaje no es otra cosa que un aproximativo juego de símbolos; para aquéllos es el mapa del universo. El platónico sabe que el universo es de algún modo un cosmos, un orden; ese orden, para el aristotélico, puede ser un error o una ficción de nuestro conocimiento parcial" (Borges, 1960, p. 96). En virtud de la clasificación de concepciones del conocimiento propuesta por Borges, este último tipo de SOC podría considerarse como una realización platónica: la idea de que existe un orden, un conjunto de clases que al ser aplicadas al mundo hacen emerger una organización coherente y total.

El uso de vocabularios controlados para la indización sigue la dinámica analítica de la construcción de conceptos científicos: introduce un objeto o referente dado dentro de un sistema de determinaciones lógicoverbales. El modelo de orden del vocabulario, la red de coherencia léxico-semántica, resulta determinante 
(Luria, 1984). Este tipo de SOC basa su condición de posibilidad en la preexistencia del modelo de orden tanto al sujeto como al objeto, es el SOC en el que lo instituido e instituyente resulta más explícito y visible.

Como se dijo anteriormente, este tipo de SOC cuenta con una tradición fundante en nuestra profesión. La posibilidad de disponer de un modelo totalizador facilita un término acariciado por la tradición bibliotecológica con cariño y devoción: la noción de "universalidad", la posibilidad de consolidar un modelo de orden y clasificación que abarque todos los fenómenos y elementos. Son ejemplos de este tipo de SOC todos los vocabularios controlados: la Clasificación Decimal Universal y la Clasificación Decimal Dewey, las listas de encabezamientos, listas de términos u ontologías.

En sus implementaciones, estos sistemas permiten vincular recursos sobre las relaciones semánticas definidas en los vocabularios. De esta manera, se realizan generalizaciones, deducciones, inducciones y otras formas de asociación entre recursos basado en el modelo de coherencias semánticas definidas para los términos. Por ejemplo, si se considera que los perros son mascotas, que el término can es un sinónimo de perro y que las veterinarias son negocios relacionados con las mascotas, un entorno de búsqueda podrá proponer explorar veterinarias toda vez que se busque perros o canes. Este tipo de funcionalidades proceden gracias al soporte de este tipo de Sistemas de representación del conocimiento.

Estos sistemas encuentran espacios favorables en entornos caracterizados por la mediación institucional (escuelas, universidades, estado, etc.) y contextos o dominios en los que se dispone de un modelo epistemológico específico, sistemáticos y explícito (dominios científicos o académicos, colegios profesionales, etc.).

\section{CONCLUSIONES}

A lo largo del presente trabajo se ha establecido un modelo orgánico e integral capaz de contener, describir, identificar y explicar una variedad de fenómenos y herramientas en términos de sistemas de organización del conocimiento. El modelo retoma líneas de pensamiento anteriores acerca de la necesidad de disponer de una teoría del significado para poder abordar la problemática de los sistemas de organización del conocimiento. En el análisis realizado se parte de la adopción de una teoría del significado para luego pasar a una teoría del conocimiento y finalmente operacionalizar conceptual y metodológicamente el marco de la organización del conocimiento.

Se ha definido la especificidad de los SOC en términos de infraestructuras sociales de mediación simbólica cuya dinámica operativa depende del modelo de autoridad cognitiva (o régimen de verdad) subyacente en cada caso. Tal como se ha señalado, en todo proceso social de mediación simbólica se recurre a un modelo de autoridad cognitiva (o régimen de verdad). En el caso de los SOC, este modelo se encuentra diseñado, formalizado y sistematizado. Se ha analizado que dicha dinámica se organiza en torno a un esquema triádico de la producción de sentido: un SOC es analizado y evaluado según sus capacidades para articular a través de elementos léxicos (L), un conjunto de conceptos (I) relativos a un conjunto de referente (R). En virtud de lo señalado, la tipología de SOC se organiza según el elemento o vértice preponderante al que apela para la consolidación de su autoridad cognitiva. De esta manera, los casos y tipos de SOC se distribuyen en un espectro tri-polar definido por la preponderancia o tipo de utilización de cada uno de los vértices. Como en cualquier espectro definido por tensiones polares, se releva un número acotado de casos puros y resultan más comunes los casos híbridos. Por ejemplo, la utilización de mecanismos de aprendizaje automático guiado por un modelo de clasificación constituye un ejemplo de hibridez entre un SOC basado en una modelización del intérprete (I) y una red de coherencias léxico-semánticas (L). Si a este SOC se le agrega una estrategia basada en procesamiento de lenguaje natural (por ejemplo: procesamiento a texto completo), se estará incorporando el tercer componente $(\mathrm{R})$ de autoridad cognitiva. En un caso de real de diseño de un SOC, restaría aún definir el rol y el peso de cada uno de los polos en el proceso de representación y búsqueda de recursos. 
Como ha podido verse a lo largo del artículo son varios los autores que consideran que la noción de significado es central para definir y analizar los SOC. Lo que el presente análisis propone es la necesidad de reconocer la existencia de diversas concepciones acerca del significado y como se materializan y proyectan en los SOC. ¿Es el significado el resultado de las relaciones entre las palabras consideradas como una red de coherencias y contrastes semánticos? ¿Es el resultado del régimen de asociación entre las palabras y sus referentes? ¿Es el resultado de los usos sociales del lenguaje? Este tipo de preguntas acerca de la concepción del significado organiza y caracteriza cada SOC.

Se ha ampliado tanto el campo de acción como los límites teóricos y metodológicos del campo de la organización del conocimiento. Un número mayor de casos y fenómenos pueden ser abordados, explicados y operacionalizados. A través del presente modelo unificado se espera dar cuenta de un territorio vasto, poblado y fértil que va desde el diseño de herramientas de búsqueda en entornos abiertos como la web hasta la formalización de dominios acotados y especializados a través de glosarios u otras herramientas terminológicas.

\section{REFERENCIAS}

Almeida, M.; Souza, R. R. \& Fonseca, F. (2011) Semantics in the Semantic Web: A Critical Evaluation. Knowledge organization, 38(3), 187-203.

Anderson, C. (2008). The end of theory: the data deluge makes the scientific method obsolete. Wired magazine. Recuperado de https://www.wired.com/2008/06/pb-theory

Barité, M. (2011). Sistemas de organización del conocimiento: una tipología actualizada. Informação \& informação, 16(2), 122-139. Recuperado de http://www.uel.br/revistas/uel/index.php/informacao/article/view/9952

Blair, D. C. (1990). Language and representation in information retrieval. Amsterdam: Elsevier.

Bliss, H. E. (1933). The organization of knowledge in libraries and the subject-approach to books. New York: Wilson.

Borges, J. L. (1960). Otras inquisiciones. Buenos Aires: Emecé.

Bowker, G. C. \& Star, S. L. (2000). Sorting things out: classification and its consequences. Cambridge: Massachusetts Institute of Technology.

Dahlberg, I. (1978). A referent-oriented, analytical concept theory for INTERCONCEPT. International classification, 5(3), 142- 151. https://doi.org/10.5771/0943-7444-1978-3-142

Dahlberg, I. (1993). Knowledge organization: its scope and possibilities. Knowledge organization: problems and tendencies, 4, 211-222.

Dahlberg, I. (2006). Knowledge organization: a new science? Knowledge organization, 33, 11-19.

Daston, L. \& Galison, P. (2007). Objectivity. New York: Zone Books.

Eco U. (1994). Signo. Barcelona: Labor.

Eco, U. (2011). El vértigo de las listas. IC - Revista cientifica de información y comunicación, 8, 15-34.

Foucault, M. (2014). Del gobierno de los vivos: curso en el Collegge de France (1979-1980). Buenos Aires: Fondo de Cultura Económica.

Glushko, R. J. (ed). (2014). The discipline of organizing. Cambridge, Massachusetts: The MIT Press.

Glushko, R. J.; Maglio, P. P.; Matlock, T., \& Barsalou, L. W. (2008). Categorization in the wild. Trends in cognitive sciences, 12(4), 129-135. https://doi.org/10.1016/j.tics.2008.01.007

Goldman, J. L. (2010). The cognitive authority of collective intelligence. (Tesis), Drexel University, Philadelphia. Recuperado de http://hdl.handle.net/1860/3254

Hjørland, B. (2007). Semantics and knowledge organization. Annual review of information science and technology, 41, 367-405.

Hjørland, B. (2008). What Is knowledge organization (KO)? Knowledge organization, 35, 86-102. 
Hjørland, B. (2012). Is classification necessary after Google? Journal of documentation, 68(3), 299-317. https://doi.o $\mathrm{rg} / 10.1108 / 00220411211225557$

Hjørland, B. (2015). Theories are knowledge organizing systems (KOS). Knowledge Organization, 42(2), 113-128.

Hodge, G. (2000). Systems of knowledge organization for digital libraries: beyond traditional authority files. Washington, DC: CLIR. Recuperado de https://clir.wordpress.clir.org/wp-content/uploads/sites/6/pub91.pdf

Kumbhar, R. (2012). Modern knowledge organisation systems and interoperability. Library classification trends in the 21st century, 95-113. https://doi.org/10.1016/b978-1-84334-660-9.50008-4

Lancaster, F. W. (1998). Indexing and abstracting in theory and practice. London: The Library Association.

Lara, M. (2015). Propostas de tipologias de KOS: uma análise das referências de formas dominantes de organização do conhecimento. Encontros Bibli: revista eletrônica de biblioteconomia e ciência da informação, 20(1), 89-107. Recuperado de http://periodicos.ufsc.br/index.php/eb/article/view/1518-2924.2015v20nesp1p89/28637

Latour, B. (1998). Visualización y cognición: pensando con los ojos y con las manos. La balsa de la Medusa, 45-46, 77-128.

Leroi-Gourhan, A. (1971). El gesto y la palabra. Caracas: Publicaciones de la Universidad Central de Venezuela.

Licklider, J. C. R. (1965). Libraries of the future. Cambridge, Mass: M.I.T. Press. Recuperado de https://archive.org /details/librariesoffutur00lickuoft

Luria, A. (1984). Conciencia y lenguaje. Madrid: Visor.

Martín Serrano, M. (1977). La mediación social. Madrid: Akal.

Martínez Tamayo, A. M. y Valdez, J. C. (2008). Indización y clasificación en bibliotecas. Buenos Aires: Alfagrama.

Mazzocchi, F. (2018). Knowledge organization system (KOS): an introductory critical account. Knowledge organization, 45(1), 54-78. http://dx.doi.org/10.5771/0943-7444-2018-1-54

Ogden, C. K. \& Richards, I. A. (1964). El significado del significado. Buenos Aires: Paidós.

Romanos de Tiratel, S. (1993). La función del bibliotecario en la sociedad del futuro. En Reunión Nacional de Bibliotecarios, Buenos Aires, Argentina.

Sartori, G. (1984). Social science concepts: a systematic analysis. London: Sage.

Sexto Empírico. (2012). Contra los dogmáticos. Madrid: Gredos.

Smiraglia, R. P. (2014). The elements of knowledge organization. Cham: Springer.

Soergel, D. (2009). Knowledge organization systems. Overview. Recuperado de http://citeseerx.ist.psu.edu/viewdoc/s ummary?doi=10.1.1.718.8120

Souza, R. R.; Tudhope, D. \& Almeida, M. B. (2012). Towards a taxonomy of KOS: dimensions for classifying knowledge organization systems. Knowledge organization, 39(3), 179-192. http://dx.doi.org/10.5771/0943-74 44-2012-3-179

Suárez Sánchez, A. (2017). Sistemas para la organización del conocimiento: definición y evolución histórica.E-Ciencias de la información, 7(2), 1-18. Recuperado de https://doi.org/10.15517/eci.v7i2.26878Foucault

Weinberger, D. (2007). Everything is miscellaneous: the power of the new digital disorder. New York: Times Books.

Wilson, P. (1983). Second hand knowledge: an inquiry into cognitive authority. Greenwood Press.

Wilson, P. (1991). Bibliographic instruction and cognitive authority. Library trends, 39(3), 259-270.

Wright, S. E. (2008). Typology for KRRs - Knowledge Representation Resources. En NKOS-CENDI Workshop. Organizado por CENDI y la Networked Knowledge Organization Systems Working Group, Washington, United States. Recuperado de https://nkos.slis.kent.edu/2008workshop/SueEllenW right.pdf

Zeng, M. L. (2008). Knowledge organization systems (KOS). Knowledge organization, 35(2-3), 160-182. http://dx.d oi.org/10.5771/0943-7444-2008-2-3-160 


\section{Notas}

1 Traducción propia.

2 Traducción propia.

3 Término acuñado primeramente en la conferencia de la ACM Digital Libraries, 1998, Pittsburgh, Pennsylvania (Hodge, 2000).

4 Traducción propia.

5 "Pero, aunque este ha sido el principal punto de desacuerdo respecto a la verdad, ha habido también entre estos filósofos otra controversia, según la cual unos han situado lo verdadero y lo falso en el terreno del significado, otros en el de la expresión, y otros en el de la actividad del pensamiento. De la primera opinión han sido sin duda exponentes destacados los estoicos, al afirmar que hay tres elementos que están interrelacionados: el significado, el significante y el objeto, y que, de estos, el significante corresponde a la expresión, por ejemplo el nombre «Dión»; el significado, a la propia cosa indicada por la expresión y que nosotros percibimos al someterse a nuestra facultad intelectiva, pero que los bárbaros no captan por más que oigan la expresión; y el objeto, al referente externo, como es el propio Dión” Sexto Empírico. Contra los lógicos II, 232-234. En: Contra los dogmáticos.

6 Traducción propia.

7 https://artsandculture.google.com/color 